\title{
Prevalence and associated factors of musculoskeletal disorders among working adults in Ethiopia: a systematic review protocol
}

Tsiwaye Gebreyesus ( $\sim$ nadaeyoba@gmail.com )

Mekelle University College of Health Sciences https://orcid.org/0000-0003-3416-5463

Balamurugan Janakiraman

University of Gondar College of Medicine and Health Sciences

Kalkidan Nigussie

University of Gondar College of Medicine and Health Sciences

\section{Protocol}

Keywords: Prevalence, Work-related musculoskeletal pain, Population-based observational studies, Systematic review, Ethiopia

Posted Date: January 17th, 2020

DOI: https://doi.org/10.21203/rs.2.21204/v1

License: (a) (1) This work is licensed under a Creative Commons Attribution 4.0 International License. Read Full License 


\section{Abstract}

Background: Musculoskeletal disorders among the working population are a major public health problem, resulting in significant personal and national economic loss. However, in Ethiopia, the cloud of infectious and non-communicable diseases has led to a lack of attention to work-related musculoskeletal disorders and empirical under-representation. This systematic review and meta-analysis will provide the pooled prevalence of musculoskeletal disorders and its determinant factors among the adult working population in Ethiopia.

Method: The electronic databases MEDLINE, PubMed, CINAHL, Science Direct index, SCOPUS, PEDro, and Google Scholar will be systematically searched. In addition, the grey literature resources such as databases or websites of dissertations and theses will be searched. The reference list of screened articles will also be hand searched. All observational studies reporting on the prevalence of work-related musculoskeletal pain of any bodily region among adult Ethiopians will be included. Random and quality effects models will be used to calculate pooled prevalence with a $95 \%$ confidence interval. Subgroup and sensitivity analyses will be performed. Publication bias and heterogeneity between the included studies will also be assessed and reported.

Discussion: The proposed systematic review and meta-analysis will provide valid insight into the pooled prevalence of multi-regional work-related musculoskeletal pain and factors associated to assist policymakers in occupational health and health care sectors. And further, we strongly believe that the data will also immensely help the understanding of the factors associated and in developing evidencebased musculoskeletal health promotion, work exposure implementations, and prevention programs in workplaces.

\section{Background}

Musculoskeletal disorders (MSDs) are common workplace health problems characterized by a range of symptoms like pain, ache, and discomfort in bodily regions [1, 2]. Musculoskeletal disorders (MSDs) are impairments of body structures such as muscles, tendons, fascia, ligaments, joints, nerves, bones, or a localized blood circulation system either caused or aggravated by poor fitness, and poor health habits, but a major proportion of MSDs are caused by physical work exposures [3, 4]. And, these impairment occurs secondary to sudden injuries and cumulative trauma, with the later being the most common mechanism behind work-related musculoskeletal disorders $[1,3,5]$.

Musculoskeletal disorders are the leading cause of activity limitation, productivity loss, and incur health care costs annually [6, 7]. The Burden of Diseases (BoD) and Global Burden Diseases (GBD) 2010 reported that $11 \%$ of the disease burden of developing countries are due to musculoskeletal disorders only next to communicable and non-communicable diseases (NCDs). Moreover, musculoskeletal disorders being three of the top 10 conditions in terms of disability and burden from NCDs in developing 
countries. Most particularly, LBP caused the highest disability-adjusted life years and 4th highest burden of all the NCDs in the developing countries [8-10].

Despite the GBD 2010 and WHO reports on the growing burden of work-related musculoskeletal disorders and attention needed [10]. Work-related musculoskeletal disorders and other musculoskeletal conditions still remain less prioritized and empirically unrepresented in low-middle income countries (LMICs), particularly in Ethiopia due to focus on more pressing and life-threatening health issues like NCDs and infectious diseases [11]. However, musculoskeletal disorders remain a major global health concern and an immense burden for LMICs like Ethiopia where health budgets are already constrained and channeled life-threatening conditions. Studies have shown that musculoskeletal pain prevalence among the working population in Ethiopia varies from 35-74.5\% [12-19].

Though several individual studies have reported the prevalence of regional pain, general WRMSDs and factors associated with the working Ethiopian population, to our knowledge there is no published systematic review and meta-analysis that explains pooled estimates of musculoskeletal disorders related to work and its associated factors. The data from this paper will help occupational health-related policymakers, health care professionals and programme managers in developing countries in particular Ethiopia in building better evidence-based occupational musculoskeletal health and disorders prevention programmes. Therefore, the objective of this systematic review and meta-analysis is to review the existing literature, with the objective of quantifying the burden of musculoskeletal disorders and associated factors among the working adult population in Ethiopia.

\section{Methods}

This systematic review and meta-analysis have been registered at the international prospective register of systematic review and meta-analysis (PROSPERO). This review protocol adheres to the Preferred Reporting Items for Systematic Reviews and Meta-analysis Protocols (PRISMA-P, 2015) [20,21] (Additional file 1). PRISMA flow diagram will be presented to describe the screening and selection processes. The findings of the review will be illustrated through figures and tables.

\section{Data sources and search strategies}

A systematic search of databases like MEDLINE, PubMed, CINAHL, Science Direct index, and Google Scholar will be conducted without any time restriction and in accordance with a detailed search strategy. Both Medical Subject Headings (MeSH) and free text words will be used while searching for relevant articles. We will use the following search terms; "work-related musculoskeletal disorders" [-MeSH], "musculoskeletal pain" [-MeSH], "musculoskeletal disorders" [-MeSH], neck pain, spinal pain, back pain, low back pain, shoulder pain, elbow pain, groin pain, knee pain, ankle pain in combination with "workers", "employees" "staffs", adults, "occupation", "workplace" "prevalence" and "Ethiopian", "AND" and "OR" Boolean operator terms will be used as appropriate. The search query and details like time, date of search, terms used, and the number of hits/results will be recorded for every session to keep a note new studies add during the review period. The details of electronic search terms and strategies are included in 
the supplementary information (Additional file 2). Furthermore, we will also search grey literature resources such as a database or website of dissertations and theses, google, WHO websites and websites of other professional bodies(eg; Centers for Disease Control and Prevention, Workplace Health Promotion). The reference lists of the included articles will be hand-searched for additional eligible studies. Moreover, the corresponding authors will be contacted by mail whenever a need arise or for any difficulties faced during data extraction.

\section{Inclusion and exclusion criteria}

All observational studies reporting on the prevalence of musculoskeletal disorders and/or musculoskeletal pain with factors associated with musculoskeletal disorders among the adult working population of Ethiopia will be included. Article that meets the following criteria will be included: (1) Conducted solely among adult men or women Ethiopian those who work in governmental sectors or private sectors or self-employed, (2) reported the prevalence using standardized pain scales or questionnaires (eg: Standardized Nordic Questionnaire, Dutch Musculoskeletal questionnaire, or Visual Analogue Scale) providing sufficient information to calculate prevalence, and (3) Published in English language. Review, clinical commentary, conference abstracts, letter to editors, non-human articles and studies conducted outside of Ethiopia will be excluded. Studies published in non-English language will be excluded as we do not possess resources to pay or delegate volunteers to translate the data.

\section{Study screening and selection for inclusion in the review}

The titles and abstracts of articles retrieved from the search of different databases will be stored and managed in a Zotero version 5 reference manager. Two reviewers (BJ and KN) will independently review the titles and abstract part of all the articles and case of conflict or disagreement will be solved by discussion.

\section{Data extraction and management}

Once eligible studies are identified, two independent reviewers (BJ and KN) will extract the data using a prepared standardized data extraction form. Data such as first author's last name, year of publication, study location within Ethiopia, sample size, response rate, reason for non-response, number of events, regions of pain reported,data on prevalence, recall period, ascertainment of outcome measures, risk factor or protective factors determined by each study along with their respective odds ratio (OR) and 95\% confidence interval, and information needed for the risk of bias assessment will be extracted.

\section{Risk of bias and quality assessment}

Two review authors (BJ and $\mathrm{KN}$ ) will independently assess the quality of all included studies using the Newcastle-Ottawa Quality Assessment tool adapted for cross-sectional studies [22,23]. The tool will be further adapted for use in this review (Additional file 3). Discrepancies of aggregate or total scores will be resolved by the third reviewer (TG) after a detailed evaluation of the source of the discrepancy. The tool contains three domains; selection of participants ( 3 items), quality of data (4 items), and definition of 
work-related musculoskeletal disorders (3 items). For the purpose of this review, all the items in the appraisal tool will be equally weighted and so the total score will be 10 . There will be no subminimum score criteria for inclusion of studies.

\section{Data synthesis}

The prevalence rate, logarithm of prevalence, and standard error (SE) of the logarithm of prevalence will be computed. Correspondingly, for the factors associated, the logarithm of OR and SE of the logarithms of OR will be calculated. The pooled prevalence (proportion) of musculoskeletal disorders or musculoskeletal pain-related work and the pooled odds ratios (OR) of associated factors with a 95\% confidence interval will be calculated using random-effects and quality effects models. The qualityeffects meta-analysis [24] will be used to examine how the quality of each study influenced the pooled estimate compared with the results from the random effects [25]. The quality scores of each included study will be incorporated in the calculation of study weight to improve the robustness and help minimize the estimator variance and subjectivity in quality assessment. The presence of heterogeneity among studies will be examined using both Cochrane's Q statistics and the ${ }^{2}$ statistics. $P$ values greater than $50 \%$ will be declared as the presence of substantial heterogeneity $[26,27]$. Funnel plots will be used to assess publication bias[28] and in addition Egger's regression test $(p<0.05)$ [29] and Beggs equations will be computed to declare publication bias. Double arsine transformation will be used in case of variance instability [30].

Possible subgroups will be identified based on the study characteristics and population characteristics. Subgroup analysis will be performed to determine the source of heterogeneity attributed to gender, sample size, place of study, study setting, year of publication, outcome tool used, type of occupation, and region of pain. Sensitivity analysis will be performed after excluding each study one by one and the pooled estimate will be calculated for the remaining studies. All statistical analyses will be performed using Meta XL version 5.3 [31] and STATA 15 Metaprop package [32].

\section{Presenting and reporting of the results}

The study selection step by step process will be outlined in a flow diagram and the reasons for exclusion will also be mentioned. The study characteristics, risk of bias, and quality assessment of the included studies will be presented in tables. Forest plots will be used to display the pooled estimates of prevalence proportions.

\section{Discussion}

This will be the first systematic review and meta-analysis that will determine the pooled prevalence of musculoskeletal disorders among the adult working population in Ethiopia. In addition, this paper will also identify factors associated with work-related musculoskeletal disorders among Ethiopians. Though WRMSD is not life-threatening, initiatives to include WRMSDs is critical in developing countries rather 
than being stand-alone. We believe that the inclusion of WRMSDs in Ethiopia will avoid efforts doubling and wasting of resources in the future.

\section{Conceivable Limitations}

We anticipate that publication bias and heterogeneity may pose a limitation for this review. There are also possibilities of reporting and response set bias.

\section{Declarations}

\section{Ethical approval and consent to participate:}

Not required

\section{Consent for publication:}

Not applicable

\section{Data availability:}

Not applicable

\section{Competing interests:}

The authors declare no competing interests

\section{Funding:}

The authors have not declared a specific grant for this review from any funding organization or agency.

\section{Authors contributions}

TG conceived the research question, edited the protocol, and designed the study, $\mathrm{KN}$ and BJ designed the search strategy, will participate in the search process, appraise the quality of the articles, and extract needed data independently. TG will analyze and interpret the results. All authors read and approve this protocol prior to sending for the publication. TG will be the guarantor and corresponding person on behalf of all the reviewers.

\section{Acknowledgments:}

We thank Mekelle University, School of Medicine and University of Gondar, School of Medicine for their help in access to University subscribed web databases.

\section{Authors information}


TG is working as a Lecturer of Physiotherapy, School of Medicine, College of Medicine and Health Sciences, Mekelle University, Mekelle, Ethiopia. BJ Assistant professor of Physiotherapy \& Senior clinical consultant physiotherapist and KN lecturer in Physiotherapy, Senior Physiotherapist, School of Medicine, College of Medicine and Health sciences and specialized hospital, University of Gondar, Gondar, Ethiopia

\section{Abbreviations}

BoD - Burden of Diseases, GBD - Global Burden of Disease, LBP - Low Back Pain, LMICs - Low-middle income countries, MeSH - Medical Subject Headings, MSDs - Musculoskeletal Disorders, NCD - NonCommunicable Diseases, OR - Odds Ratio, PRISMA-P - Preferred Reporting Items for Systematic Reviews and Meta-analysis Protocols, SE - Standard Error, WHO - World Health Organization, WRMSDs - WorkRelated Musculoskeletal Disorders.

\section{References}

1. Al-Hourani Z, Nazzal M, Khader Y, Almhdawi K, Bibars AR. Work-related musculoskeletal disorders among Jordanian dental technicians: Prevalence and associated factors. Work. 2017;56(4):617-23.

2. Bethge M. [Work-Related Medical Rehabilitation]. Rehabilitation (Stuttg). 2017 Feb;56(1):14-21.

3. Roquelaure Y, Bodin J, Descatha A, Petit A. [Musculoskeletal disorders: how to recognize them as occupational diseas]. Rev Prat. 2018 Dec;68(10):1132-4.

4. Gómez-Galán M, Pérez-Alonso J, Callejón-Ferre Á-J, López-Martínez J. Musculoskeletal disorders: OWAS review. Ind Health. 2017 Aug 8;55(4):314-37.

5. Oranye NO, Bennett J. Prevalence of work-related musculoskeletal and non-musculoskeletal injuries in health care workers: the implications for work disability management. Ergonomics. 2018;61(3):355-66.

6. Asfaw A, Pana-Cryan R, Bushnell T, Sauter S. Musculoskeletal disorders and associated healthcare costs among family members of injured workers. American journal of industrial medicine. 2015;58(11):1205-16.

7. Beaudart C, Biver E, Bruyère 0 , Cooper C, Al-Daghri N, Reginster J-Y, et al. Quality of life assessment in musculo-skeletal health. Aging clinical and experimental research. 2018;30(5):413-8.

8. Hoy DG, Smith E, Cross M, Sanchez-Riera L, Buchbinder R, Blyth FM, et al. The global burden of musculoskeletal conditions for 2010: an overview of methods. Annals of the rheumatic diseases. 2014;73(6):982-9.

9. Hoy DG, Smith E, Cross M, Sanchez-Riera L, Blyth FM, Buchbinder R, et al. Reflecting on the global burden of musculoskeletal conditions: lessons learnt from the global burden of disease 2010 study and the next steps forward. Annals of the rheumatic diseases. 2015;74(1):4-7.

10. Storheim K, Zwart J-A. Musculoskeletal disorders and the Global Burden of Disease study. 2014;

11. Woolf AD, Brooks P, Åkesson K, Mody GM. Prevention of musculoskeletal conditions in the developing world. Best Practice \& Research Clinical Rheumatology. 2008;22(4):759-72. 
12. Temesgen MH, Belay GJ, Gelaw AY, Janakiraman B, Animut Y. Burden of shoulder and/neck pain among school teachers in Ethiopia. BMC musculoskeletal disorders. 2019;20(1):18.

13. Kebede Deyyas W, Tafese A. Environmental and organizational factors associated with elbow/forearm and hand/wrist disorder among sewing machine operators of garment industry in Ethiopia. Journal of environmental and public health. 2014;2014.

14. Dheresa M, Geda B. Low Back Pain and Associated Factors among Nurses Working in Public Hospitals of Harari region and Dire Dawa City Administration, Eastern Ethiopia. 2018;

15. Yosef T, Belachew A, Tefera Y. Magnitude and Contributing Factors of Low Back Pain among Long Distance Truck Drivers at Modjo Dry Port, Ethiopia: A Cross-Sectional Study. Journal of environmental and public health. 2019;2019.

16. Mekonnen TH. Work-Related Factors Associated with Low Back Pain Among Nurse Professionals in East and West Wollega Zones, Western Ethiopia, 2017: A Cross-Sectional Study. Pain and therapy. 2019;8(2):239-47.

17. Wami SD, Dessie A, Chercos DH. The impact of work-related risk factors on the development of neck and upper limb pain among low wage hotel housekeepers in Gondar town, Northwest Ethiopia: institution-based cross-sectional study. Environmental health and preventive medicine. 2019;24(1):27.

18. Tafese A, Nega A, Kifle M, Kebede W. Predictors of occupational exposure to neck and shoulder musculoskeletal disorders among sewing machine operators of garment industries in Ethiopia. Science Journal of Public Health. 2014;2(6):577-83.

19. Abraha TH, Demoz AT, Moges HG, Ahmmed AN. Predictors of back disorder among Almeda textile factory workers, North Ethiopia. BMC research notes. 2018;11(1):304.

20. Moher D, Shamseer L, Clarke M, Ghersi D, Liberati A, Petticrew M, et al. Preferred reporting items for systematic review and meta-analysis protocols (PRISMA-P) 2015 statement. Systematic reviews. 2015;4(1):1.

21. Shamseer L, Moher D, Clarke M, Ghersi D, Liberati A, Petticrew M, et al. Preferred reporting items for systematic review and meta-analysis protocols (PRISMA-P) 2015: elaboration and explanation. Bmj. 2015;349:g7647.

22. Luchini C, Stubbs B, Solmi M, Veronese N. Assessing the quality of studies in meta-analyses: Advantages and limitations of the Newcastle Ottawa Scale. World J Meta-Anal. 2017;5(4):80-4.

23. The Newcastle-Ottawa scale adapted for cross-sectiona studies [https:// wellcomeopenresearch.s3.amazonaws.com/supplementary/13880/ea30a2fba15a-44a9-b35e5f0914db80b3.docx]. Accessed 1 Feb 2019.

24. Doi SA, Thalib L. A quality-effects model for meta-analysis. Epidemiology. 2008;94-100.

25. Berkey CS, Hoaglin DC, Mosteller F, Colditz GA. A random-effects regression model for meta-analysis. Statistics in medicine. 1995;14(4):395-411.

26. Higgins JP, Thompson SG. Quantifying heterogeneity in a meta-analysis. Statistics in medicine. 2002;21(11):1539-58. 
27. Stroup DF, Berlin JA, Morton SC, Olkin I, Williamson GD, Rennie D, et al. Meta-analysis of observational studies in epidemiology: a proposal for reporting. Jama. 2000;283(15):2008-12.

28. Liu JL. The role of the funnel plot in detecting publication and related biases in meta-analysis. Evidence-based dentistry. 2011;12(4):121.

29. Sterne JA, Egger M. Regression methods to detect publication and other bias in meta-analysis. Publication bias in meta-analysis: Prevention, assessment and adjustments. 2005;99-110.

30. Barendregt JJ, Doi SA, Lee YY, Norman RE, Vos T. Meta-analysis of prevalence. J Epidemiol Community Health. 2013;67(11):974-8.

31. Barendregt J, Doi S. MetaXL user guide version 5.3. EpiGear International Pty Ltd. 2016;

32. Nyaga VN, Arbyn M, Aerts M. Metaprop: a Stata command to perform meta-analysis of binomial data. Archives of Public Health. 2014;72(1):39.

\section{Supplementary Files}

This is a list of supplementary files associated with this preprint. Click to download.

- Additionalfile1.docx

- Additionalfile3.docx

- Additionalfile2.docx 